significant persistent effect on step-counts and no difference between nurse and postal group outcomes. In PACE-Lift the step-count effect, while not statistically significant was consistent with both the PACE-UP findings and with the persistent significant effect for MVPA in bouts in PACE-Lift. These findings suggest that primary care pedometer interventions, delivered by post or with minimal contact, can make an important long-term contribution to addressing the public health physical inactivity challenge.

\section{OP82 SYSTEMATIC REVIEW OF DIGITAL INTERVENTIONS TO IMPROVE THE DIET AND PHYSICAL ACTIVITY BEHAVIOURS OF ADOLESCENTS}

\begin{abstract}
${ }^{1} \mathrm{~T}$ Rose*, ${ }^{1,2} \mathrm{M}$ Barker, ${ }^{3} \mathrm{CM}$ Jacob, ${ }^{1,2} \mathrm{~W}$ Lawrence, ${ }^{1} \mathrm{~S}$ Strommer, ${ }^{1,2} \mathrm{C}$ Vogel, ${ }^{2,4} \mathrm{~K}$ WoodsTownsend, ${ }^{1,2} \mathrm{H}$ Inskip, ${ }^{1,2} \mathrm{~J}$ Baird. ${ }^{1} \mathrm{MRC}$ Lifecourse Epidemiology Unit, University of Southampton, Southampton UK; ${ }^{2}$ NIHR Southampton Biomedical Research Centre, University Hospital Southampton NHS Foundation Trust, University of Southampton, Southampton UK; ${ }^{3}$ Academic unit of Human Development and Health, Faculty of Medicine, University of Southampton, Southampton UK; ${ }^{4}$ Southampton Education School, University of Southampton, Southampton, UK
\end{abstract}

\subsection{6/jech-2017-SSMAbstracts.81}

Background Adolescents have poor diets and insufficient physical activity (PA) levels, which can lead to non-communicable diseases in later life. Adolescence is a key stage for establishment of lifelong health behaviours. Digital platforms offer inexpensive means of delivering health interventions to large numbers of adolescents, but little is known about their effectiveness in supporting sustained changes. This systematic review was conducted to synthesise evidence on the effectiveness of digital interventions to improve diet quality and increase PA in adolescents, to identify effective intervention components and to assess the cost-effectiveness of these interventions.

Methods A systematic review of digital interventions to improve the diet and/or physical activity behaviours of adolescents was carried out according to established guidance. A search of MEDLINE, PsycINFO, CINAHL, PubMed Central, Embase, ERIC, the NHS EED and CENTRAL was conducted using a combination of $\mathrm{MeSH}$ and free text terms. Abstracts were assessed by two independent researchers against review inclusion criteria (intervention studies with and without a control group, participants aged 10-19, interventions that included a digital component, studies that measured a diet or PA outcome at baseline and follow-up, any setting and any population of adolescents). For included studies, data extraction and quality assessment were performed using a form designed to capture key information to answer the research questions. We then analysed data to identify key intervention features that were associated with significant improvements in behaviour. We also aimed to collect data on cost-effectiveness of included interventions.

Results The search returned 6792 results, of which 86 full texts were selected for screening. After assessing these against eligibility criteria, 27 intervention studies were included in the review. Most $(n=15)$ were website interventions. Other delivery methods were text messages $(n=4)$, gamified interventions $(n=3)$, multi-component interventions that included a digital element $(n=3)$, email $(n=1)$, and social media $(n=1)$. Significant behaviour change was often seen when interventions included diet and physical activity education, goal-setting in conjunction with self-monitoring, and parental involvement.
None of the included publications reported cost-effectiveness. Due to heterogeneity of studies, meta-analysis was not feasible.

Conclusion It is possible to effect significant health behaviour change in adolescents through targeted digital interventions that incorporate education, goal-setting, self-monitoring and parental involvement. Most of the evidence relates to websites and further research is needed to assess effectiveness of interventions delivered via alternate media such as smartphone apps. Longer term outcomes should be evaluated. Future trials of digital interventions need to evaluate cost-effectiveness.

\section{OP83 DIET AND PHYSICAL ACTIVITY-BASED WEIGHT MANAGEMENT PROGRAMMES FOR ADULTS: RE- ANALYSIS OF A SYSTEMATIC REVIEW THROUGH THE LENS OF USER VIEWS TO IDENTIFY PATHWAYS TO EFFECTIVENESS}

${ }^{1} \mathrm{GJ}$ Melendez-Torres, ${ }^{2} \mathrm{~K}$ Sutcliffe*, ${ }^{3} \mathrm{HED}$ Burchett, ${ }^{2} \mathrm{R}$ Rees, ${ }^{2} \mathrm{M}$ Richardson, ${ }^{2} \mathrm{~J}$ Thomas. ${ }^{1}$ Warwick Medical School, University of Warwick, Coventry, UK; ${ }^{2}$ EPPI-Centre, UCL Institute of Education, London, UK; ${ }^{3}$ Policy Innovation Research Unit, London School of Hygiene and Tropical Medicine, London, UK

\subsection{6/jech-2017-sSMAbstracts.82}

Background Previous systematic reviews of weight management programmes have not been able to account for heterogeneity of effectiveness within programmes using top-down behavioural change taxonomies and standard meta-regression methods. This could be due to the complex nature of these interventions, the intersecting aspects of different intervention components, and the presence of overlapping causal pathways to effectiveness (or lack of effectiveness). Qualitative comparative analysis is a method that, when used in the synthesis of complex interventions, can help identify the 'how' and 'why' of intervention effectiveness while accounting for these overlapping and intersecting causal pathways.

Methods We updated a previous systematic review of studies relating to the views of programme users and trials of weight management programmes in adults that included dietary and physical activity components. We thematically analysed views studies to identify intervention features perceived to be important by service users and providers. We selected the most and least effective trials as measured by amount of weight loss at 12 months compared to minimal treatment. Using intervention components suggested by the synthesis of user views, we labelled interventions as to the absence or presence of these components and, using qualitative comparative analysis, we identified combinations of components that created the conditions sufficient for interventions to be most effective and least effective.

Results Our analysis included 21 studies of user views reported in 26 papers, and 20 interventions - 10 most effective and 10 least effective-reported in 15 trials. The synthesis of user views suggested three facets of interventions for analysis: provider-user alliance, or the quality of the relationship between users and providers; provider directiveness, or the perceived high need for guidance from providers; and group relationships, or the quality of the relationship between peers in weight management programmes. Across our analyses, we found that pathways to intervention effectiveness were characterised by the presence of opportunities to develop supportive relationships with providers or peers, clear direction from 
providers together with components to develop self-regulation of exercise and dietary behaviour.

Discussion Revisiting complex interventions through a lens that accounts for complexity can facilitate action on a pressing public health problem like obesity and overweight. Though QCA is an inductive method, this innovative approach has enabled the identification of neglected critical aspects of WMPs, such as the nature of relationships within them, which were previously not considered to be as important as more concrete content such as dietary focus.

\section{OP84 ARE WE THERE YET? A CUMULATIVE META-ANALYSIS OF THE ACCUMULATION OF EVIDENCE FOR PHYSICAL ACTIVITY PROMOTION}

\begin{abstract}
${ }^{1,2}{ }^{2}$ Love*, ${ }^{1} E$ van Sluijs, ${ }^{11}$ Adams, ${ }^{2}$ D Humphreys. ${ }^{1}$ Centre for Diet and Activity Research (CEDAR), MRC Epidemiology Unit, University of Cambridge School of Clinical Medicine, Cambridge, UK; ${ }^{2}$ Department of Social Policy and Intervention, University of Oxford, Oxford, UK; ${ }^{3}$ Department of Social Policy and Intervention, University of Oxford, Oxford, UK
\end{abstract}

\subsection{6/jech-2017-SSMAbstracts.83}

Background Despite a large evidence base and increasing number of physical activity interventions, rates of physical inactivity and associated chronic diseases are rapidly increasing globally. It is an imperative time to examine if new intervention trials have contributed novel evidence to the field. The purpose of this overview of systematic reviews is to investigate the evolution of evidence for physical activity promotion interventions over time through the analysis of changes in effect sizes for physical activity (PA) and cardiorespiratory fitness (CR) outcome measures.

Methods The search process was conducted in two stages. In the first phase, the Cochrane Central Register of Controlled Trials, MEDLINE, EMBASE, CINAHL, PsycINFO and Web of Science were searched for systematic reviews that examined the effects of interventions targeted at increasing physical activity within individuals, and included randomised controlled trials. In the second phase, primary studies included within the eligible systematic reviews were pooled. Interventions aiming to increase physical activity in healthy adults at the individual level, assessed through randomised controlled trials, were included. A cumulative meta-analysis was performed separately for interventions with PA and CR outcome measurements.

Results When assessed through cumulative meta-analysis, the totality of the evidence demonstrates that intervention effectiveness has not changed very much over the past fifteen years and that further trials are unlikely to overturn the positive and stabilised findings. The analyses indicate that the effect size for interventions became precise and stable in 2001 after the conduct of 12 physical activity (PA) and 11 cardiorespiratory (CR) outcome trials. In the time following the attainment of these thresholds of stability and precision, 70 further full scale RCTs (59 for PA and 11 for CR outcomes) have been conducted.

Conclusion Substantial evidence exists demonstrating that physical activity interventions can modify individual behaviour. However, there is limited evidence of advancements in intervention effectiveness. Given the stability and sufficiency of the evidence base, researchers are urged to shift focus towards the optimisation and scaling-up of interventions across populations.

\section{Methods}

\section{OP85 A QUALITATIVE EXPLORATION OF TRIAL-RELATED TERMINOLOGY IN A STUDY INVOLVING DEAF BRITISH SIGN LANGUAGE USERS}

${ }^{1} \mathrm{~A}$ Young ${ }^{*},{ }^{1} \mathrm{C}$ Dodds, ${ }^{1} \mathrm{R}$ Oram, ${ }^{1} \mathrm{C}$ Nassimi-Green, ${ }^{1} \mathrm{R}$ Belk, ${ }^{1} \mathrm{~K}$ Rogers, ${ }^{2} \mathrm{~L}$ Davies, ${ }^{3} \mathrm{~K}$ Lovell. 'Social Research with Deaf People, School of Health Sciences, University of Manchester and MAHSC, Manchester, UK; ${ }^{2}$ Health Services Research and Primary Care, Institute of Population Health, University of Manchester and MAHSC, Manchester, UK; ${ }^{3}$ Division of Nursing, Midwifery and Social Work, University of Manchester and MAHSC, Manchester, UK

\subsection{6/jech-2017-SSMAbstracts.84}

Background British Sign Language (BSL) is a fully grammatical, living language, independent of spoken English. Deaf BSL users are routinely excluded from clinical trials either on grounds of language and/or assumptions of confounding factors associated with disability. Few clinical trials specifically targeted at Deaf people exist internationally. Appropriate terminology in BSL for key concepts in clinical trials is needed to support informed consent.. Barriers to conceptual (mis) understanding of trial participation and sources relevant to the Deaf community are unexplored. This study set out to:

(i) Explore, in BSL, the meaning and understanding of key concepts and common vocabulary used in recruitment and informed consent to a clinical trial

(ii) Enable signs/signed expressions to emerge that are semantically accurate and support Deaf people's informed consent in any future trial

Methods A community participatory approach underpinned data collection. This comprised Deaf-led, qualitative, exploratory group discussions (19 self-selected participants in five groups), facilitated in BSL. Discussion was structured to explore the meaning of key terminology (such as trial, randomisation, consent) and through that exploration generate signed explanations of concepts to use in future trials. Data were video-recorded and analysed in BSL, using a phenomenological approach, built from the application of 4 themes and 8 sub-themes using NVIVO8 for visual data.

Results All participants were over 30 years old, with a selfdeclared 'strong Deaf identity'; BSL was their first or preferred language. Six necessary conditions for developing optimal trial information were identified. (i) developing appropriate expressions and terminology from a community basis, rather than testing out previously derived translations from a different language; (ii) paying attention to languagespecific features which support best means of expression (in the case of BSL expectations of specificity, verb directionality, handshape); (iii) accounting for bilingual influences on comprehension; (iv) deliberate orientation of information to avoid misunderstanding not just to promote accessibility; (v) sensitivity to barriers to discussion about intelligibility of information that are cultural and social in origin, rather than linguistic; (vi) the importance of using contemporary language-in-use, rather than jargon-free or plain language, to support understanding.

Conclusion These conditions need to be met to develop signed participant information for Deaf people that is: acceptable, accessible, transmitted accurately and understood as intended. They are required to address the cultural preferences and lower background knowledge of Deaf people (using signed languages) as well as in spoken/written languages when 